\title{
Des villes communistes face à l'éducation prioritaire, entre engagement marqué et refus (1981-1984)
}

\author{
Lydie Heurdier \\ Centre interdisciplinaire de recherche culture, éducation, formation, travail - \\ Éducation scolarisation \\ CIRCEFT-ESCOL, ÉA 4384 \\ Université Paris 8 Vincennes-Saint-Denis \\ lheurdier@gmail.com \\ Philippe Bongrand \\ Laboratoire École, Mutations, Apprentissages \\ ÉMA, ÉA 4507 \\ CY Cergy Paris Université \\ philippe.bongrand@cyu.fr
}

Résumé : Contrairement à une opinion commune, le Parti communiste français a bien encouragé la mise en place de la politique d'éducation prioritaire créée par le ministre socialiste Alain Savary en 1981-1982. Après l'avoir démontré à partir de la revue officielle du parti, L'école et la nation, l'article analyse la mise en œuvre de cette politique par trois municipalités communistes ayant adopté des postures contrastées. L'étude d'Aubervilliers, Le Havre et Orly, à partir de procès-verbaux de conseils municipaux, de la presse municipale et de témoignages, montre que les relations entre les élus locaux et les enseignants y ont été décisives, les élus disposant en outre d'une large autonomie.

Communist Towns and Priority Education Zones: Caught Between Clear Commitment and Refusal (1981 - 1984)

Abstract : Contrary to a common opinion, the French Communist Party encouraged the setting up of the socialist minister Alain Savary's policy of priority education zones. This paper shows this first through a study of the party's official news magazine, L'école et la nation (Our Schools and the Nation), and then focuses on the way this policy was implemented in three different and contrasting communist towns. An overview of Aubervilliers, Le Havre and Orly based on the minutes of town hall meetings, the local press and different interviews shows that the relations between local elected officials and teachers were essential to the process, elected officials benefitting furthermore from considerable autonomy. 
En 1981, la création par le nouveau ministère de l'Éducation nationale de zones prioritaires (ZP) ${ }^{1}$ prend de court les acteurs du système éducatif. Les débats précédant la victoire électorale de la gauche n'avaient pas attiré l'attention des enseignants, parents d'élèves, élus ou syndicats sur le principe d'affectation de moyens supplémentaires aux territoires concentrant le plus de difficultés sociales principe alors désigné par les expressions «école inégalitaire» ou «programmes d'éducation prioritaires $»^{2}$. Or la discrimination positive représente une « révolution conceptuelle » (Prost, 2013, p. 197) dont l'endossement et la mise en œuvre ne vont pas de soi. Certes, au sein du cabinet du nouveau ministre de l'Éducation nationale, Alain Savary, le dossier est porté par deux militants qui y réfléchissent de longue date. Christian Join-Lambert, conseiller à la Cour des Comptes, et Jean-Louis Piednoir, maitre-assistant en mathématiques, ont été membres de la Délégation à l'Éducation du Parti socialiste (PS). Désormais en situation de la mettre en œuvre, ils en connaissent les difficultés « techniquement et au fond $»^{3}$ et, pour les surmonter, ils peuvent a priori compter sur le concours des réseaux socialistes locaux et nationaux. En revanche, les partenaires communistes de la coalition gouvernementale n'ont pas endossé jusqu'alors l'idée de zones prioritaires. Leurs plates-formes électorales évoquent la réforme d'ensemble du système éducatif et l'égalité de moyens, non la discrimination positive. Or compte tenu de l'implantation du Parti communiste français (PCF) dans des communes comprenant des quartiers particulièrement défavorisés, le soutien communiste à la mesure et à sa mise en œuvre, incertain, apparaît nécessaire.

Lorsqu'elles portent sur l'échelon national, les recherches sur l'histoire de la politique d'éducation prioritaire n'abordent pas le positionnement communiste, l'attention étant centrée sur le PS (Bongrand, 2009 ; Heurdier, 2014). Les études des syndicats enseignants suggèrent que ceux proches du PCF ont minoré l'originalité et l'intérêt d'une mesure venue du SGEN (Robert, 2002). Par ailleurs, les travaux plus centrés sur l'échelon local ne se préoccupent pas de l'implication des élus et municipalités, privilégiant les personnels administratifs ou enseignants (Heurdier, 2008 ; Robert, 2009 ; Llobet, 2012). Dès 2002 pourtant, Kherroubi et Rochex signalaient la faible prise en compte du rôle des villes et, s'appuyant sur les travaux d'Agnès van Zanten, relayaient le constat, contre-intuitif, que des municipalités communistes auraient été initialement réservées à l'égard de l'éducation prioritaire (Kherroubi, Rochex, 2002, p. 121-122). Étudier ici l'attitude du PCF, aux échelons national et local, revient ainsi à aborder un angle mort de l'historiographie de l'éducation prioritaire (Bongrand, 2013).

Le présent article se concentre sur la période de mai 1981 à juillet 1984, pendant laquelle quatre ministres communistes participent au gouvernement d'union de la gauche. Tout d'abord, la position officielle nationale du PCF est présentée telle qu'elle apparaît dans sa revue éducative, L'École et la nation. Le PCF alors solidaire du gouvernement adopte une position favorable vis-à-vis de la politique des ZEP, et l'évocation, secondaire, de ses limites théoriques et pratiques vient moins disqualifier qu'assumer cette politique. L'article examine ensuite l'attitude de trois municipalités communistes. La comparaison met en évidence leurs implications contrastées. N'ayant créé aucune zone prioritaire malgré des indicateurs sociaux y engageant, Aubervilliers apparaît à l'opposé d'Orly, fortement engagée dès 1981. Le Havre présente une configuration intermédiaire, soutenant la zone sans chercher à l'orienter. Pour étudier ces trois municipalités, nous nous appuyons sur des sources multiples :

\footnotetext{
${ }^{1}$ Lors de leur création, ces zones s'intitulent officiellement « zone prioritaire » (ZP), malgré les usages, avérés dès l'amont, et par la suite durablement repris par la réglementation, du sigle «ZEP » (zone d'éducation prioritaire). Dans ce texte, qui n'aborde pas les connotations différentes - et d'ailleurs rarement débattues à l'époque - de ces deux expressions, nous les utilisons indifféremment.

${ }^{2}$ Libérer l'école : Plan socialiste pour l'Éducation nationale, Paris, Flammarion, 1978.

${ }^{3}$ Témoignage de C. Join-Lambert (in) Bourgarel et al., 2006, p. 46.
} 
débats en conseil municipal, traces du fonctionnement des ZEP, témoignages oraux, presse municipale.

Ces trois exemples introduisent à une gamme d'attitudes des élus du PCF. La mise en œuvre de la politique des ZEP ne s'avère pas exclusivement une affaire de positionnement idéologique, les communistes étant en phase avec le discours national, mais de relations, manifestement variables suivant les territoires, entre municipalités et enseignants. L'échelon local des politiques d'éducation prioritaire reste à investir par la recherche ${ }^{4}$.

\section{«Positif et loyal » : la ligne officielle du PCF en faveur des ZEP (1981-1984)}

Au regard de ses positions pendant la campagne électorale, le PCF paraît prédisposé à recevoir positivement la création des zones prioritaires. En mai 1980, dans un numéro consacré aux inégalités, l'École et la nation dénonce le traitement inégalitaire des quartiers populaires (enseignants moins formés, taux d'encadrement plus défavorables, moindres taux de réussite, offre d'options moins ample). Donner la priorité à ces territoires - intéressant le PCF notamment au plan électoral - est alors explicitement envisagé : " 25 élèves par classe, oui, mais pourquoi pas avant tout dans telle classe à forte population ouvrière, à forte proportion d'immigrés, à fortes difficultés scolaires, pourquoi pas dans tel quartier d'Aubervilliers avant telle école du $16^{e}$ arrondissement? Se battre pour les remplacements d'enseignants malades, oui, mais pourquoi pas en priorité là où les élèves sont déjà en forte situation d'échec, c'est-à-dire plutôt dans telle cité HLM de Denain plutôt que dans les beaux quartiers de Lille? Non seulement on constate que la division en classes de la société reproduit l'École mais que les privilèges des classes dominantes se retrouvent dans un système scolaire inégalitaire, inégalitaire non pas fatalement et abstraitement mais "inégalement inégalitaire" d'un point de vue de classe ${ }^{5}$. Au printemps suivant, le tract électoral pour la campagne présidentielle propose d'"orienter les enseignants les mieux qualifiés vers les écoles des quartiers les plus populaires [et de] mettre en œuvre des programmes d'urgence d'équipements et d'activités éducatives, sportives et culturelles, en commençant par les quartiers populaires».

À partir de la rentrée 1981, la revue assume un rôle inédit de soutien de la majorité gouvernementale. Elle se donne pour mission de contribuer à «la mise en route du changement, (...) [s'efforçant] d'être plus et mieux l'outil efficace qu'attendent, dans cette période à la fois exaltante et complexe, tous les militants de l'école». Commentant les premières mesures du gouvernement, la revue "retrouve dans la volonté de créer les ZEP une des constantes de [sa] démarche " et la "salue comme très positive ${ }^{6}$.

Cependant, la revue se fait rapidement l'écho d'insatisfactions. Si les ZP font la couverture du numéro d'octobre 1981, l'enquête sur la ZP de Jarville, près de Nancy, manifeste les écarts entre doctrine et pratique. Elle rapporte que, loin de la logique partenariale d'ouverture, c'est l'Éducation nationale qui y a unilatéralement identifié le territoire prioritaire, et que c'est le chef d'établissement, peu confiant dans les enseignants, qui a orienté l'usage des moyens supplémentaires vers des réductions d'effectifs par classe, alors que certains souhaitaient du temps de concertation. Suggérant que le cas est

\footnotetext{
${ }^{4}$ C'est en partie l'objet du projet en cours ARCHI-SHEP (Archives pour une socio-histoire de l'éducation prioritaire), https://archiveszep.hypotheses.org.

${ }^{5}$ Gilles Romano, «Inégalité dans les inégalités », L'école et la nation, $\mathrm{n}^{\circ}$ 302, mai 1980, p. 8-9.

${ }^{6}$ Ange-Marie Filippi-Codaccioni, «Trouver des solutions nouvelles », L'école et la nation, $\mathrm{n}^{\circ}$ 322, octobre 1981, p. 2-3.
} 
représentatif, la revue cite Christian Join-Lambert, qui émet le même constat : dans l'urgence de la mise en œuvre "on a tenu les enseignants à l'écart de la concertation sur ces zones prioritaires». L'insatisfaction relative au périmètre et aux modalités de la concertation apparaît également dans le témoignage du président de la FCPE de Seine-Saint-Denis, dont on peut supposer qu'il est communiste. C. Roulette, après avoir indiqué que les ZP reprennent des idées du PCF puis énuméré les raisons pour lesquelles la mesure a pu ne pas immédiatement convaincre, dénonce : "il faut se mettre en guerre contre ceux qui, à l'étape difficile que nous amorçons, plaident le refus, l'expectative ou la dérision ${ }^{7}$. Lors des débats budgétaires, le député PCF Guy Hermier, membre du bureau politique, réitère également le soutien aux ZP tout en réclamant plus de concertation ${ }^{8}$. Si la revue mentionne systématiquement les ZP parmi les actions positives des premiers mois du gouvernement ${ }^{9}$, c'est dans un discours nuancé, où le soutien de principe est mêlé à l'appel à améliorer la mise en œuvre, notamment en associant mieux élus, enseignants et autres partenaires, en suivant une approche moins « technocratique ».

La revue accueille dès lors très favorablement la circulaire qui, en décembre 1981, organise le travail d'élaboration d'une nouvelle carte des zones prioritaires pour la rentrée 1982: "à la lecture de cette circulaire, les zones prioritaires nous apparaissent à la fois comme la locomotive et le laboratoire de la rénovation du système éducatif. (...) l'idée que nous avions lancée de prévoir pour ces zones prioritaires un projet spécifique élaboré par tous les partenaires de l'école (personnels de l'Éducation nationale, usagers, élus locaux, syndicats et les différentes instances compétentes) est reprise par le ministère ${ }^{10}$. Le PCF, s'il reconnaît "les limites que le suffrage universel a fixées [à son poids] dans la majorité et à sa capacité d'intervention sur les décisions à prendre », se veut " positif et loyal »"

Ainsi, suivant son intérêt, qui conduisait à investir une politique qui légitimait l'action publique en territoire populaire, ouvrait l'école aux élus locaux et permettait de militer auprès des enseignants, le PCF a appelé à amplifier la politique des ZEP, déplorant au contraire qu'elle puisse rencontrer des freins - identifiés aux cadres administratifs en place, rétifs au changement (voire "serviteurs zélés » des ministres antérieurs) $)^{12}$. Lorsqu'il apparaitra en 1984, que «Le découragement a pris le pas sur l'enthousiasme ", les ZEP s'étant avérées "latérales par rapport à la question centrale du sens et de la nature de l'échec scolaire ${ }^{13}$, c'est une relance des ZEP que Guy Hermier réclamera ${ }^{14}$. Dans un contexte d'incitations convergentes à la déconcentration et à la décentralisation, comment les élus locaux ont-ils pris parti ? Examinons le cas de trois communes dont l'implication dans la politique ZEP fait contraste.

\section{Aubervilliers : ignorer les ZEP}

\footnotetext{
${ }^{7}$ Roulette C. (1982). Zones prioritaires : un parent s'explique. L'école et la nation, $\mathrm{n}^{\circ} 325$, p. 38-39.

${ }^{8}$ De l'héritage au renouveau. Propositions des députés communistes dans le débat pour le budget de l'Éducation nationale. L'école et la nation, $\mathrm{n}^{\circ} 324$, décembre 1981, p. 16-22.

${ }^{9}$ Par exemple : En vingt mois, des transformations positives. L’École et la nation. Actualités, $\mathrm{n}^{\circ} 16$, février 1983 , p. 2 ; Descottes C. (1982). Les circulaires du changement. L'École et la nation, $\mathrm{n}^{\circ} 327$, p. 31-33.

${ }^{10}$ Coulbaut C. (1982). Zones prioritaires, une circulaire. L'école et la nation, $\mathrm{n}^{\circ} 325, \mathrm{p} .4$.

${ }^{11}$ Conférence de presse de Guy Hermier. L’École et la nation. Actualités, n¹4, mai 1982, p. 2.

${ }^{12}$ Maurice R., Delfaut D. (1982). Zones prioritaires : pour avancer. L'école et la nation, ${ }^{\circ}$ 328, p. 15-20.

13 Davisse A. (1985). Pour la relance d'une politique de lutte contre l'échec scolaire et la réussite de tous. Rapport introductif. L'École et la nation, $\mathrm{n}^{\circ} 355$, p. 43

${ }^{14}$ ibid, p. 11.
} 
Limitrophe du $19^{\mathrm{e}}$ arrondissement de Paris, Aubervilliers figure parmi les villes les plus pauvres d'Îlede-France. En 1982, elle présente les caractéristiques attendues pour une ZP : fort taux de retard de deux ans et plus à l'entrée en $6^{\mathrm{e}}(25 \%)$, pourcentage élevé d'élèves de nationalité étrangère au collège $(28 \%)$. La municipalité conduit une politique culturelle ambitieuse, d'envergure nationale, et a développé des services sociaux et de santé, des équipements sportifs. La politique de l'enfance et de la jeunesse y est une priorité et les écoles bénéficient de structures gratuites et d'intervenants municipaux. D'abord adjoint puis maire de la ville (1984-2003), Jack Ralite, est ministre de juin 1981 à juillet $1984^{15}$. Aucune ZP n'est pourtant créée à la rentrée 1982, ce que le recteur justifie par l'absence de « mobilisation sur le terrain des acteurs du système éducatif, telle qu'elle s'est concrétisée notamment par les projets pédagogiques ${ }^{16}$ devant associer primaire et secondaire. La priorité a été donnée aux territoires engagés dans des projets collectifs interdegrés et partenariaux. Si à Aubervilliers aucun établissement n'a été classé avant 1998, les écoles de La Courneuve, ville communiste voisine, ont été, elles, quasi intégralement classées dès 1982.

Rétrospectivement, l'élue adjointe à l'enseignement ${ }^{17}$ déplore les relations compliquées avec les enseignants des écoles : difficulté à obtenir des données statistiques fines, réticence enseignante à cibler les élèves lors de certains projets, existence de projets pédagogiques peu élaborés, absence de vraies discussions dans les instances comme les conseils d'école. L'opposition «farouche»du directeur d'une école primaire, sa peur du partenariat, auraient repoussé à 1998 le classement de cette école. Les élus, eux, auraient été en 1981 favorables à la démarche : "on avait été sensible à des moyens inégalitaires pour des situations inégalitaires ». Si en 1983-1984, les démarches auprès de l'inspection académique pour obtenir le classement n'ont pas abouti, officiellement faute de moyens suffisants, l'hypothèse d'un positionnement divergent entre élus et enseignants est partiellement convaincante.

Car la consultation des procès-verbaux de conseils municipaux (CM), pour la période 1981-1985, interroge : il ne fut jamais question, dans les débats en son sein, de la politique d'éducation prioritaire. En 1981 et 1982, le CM n'aborde la question scolaire que sous l'angle des travaux à effectuer ou du fonctionnement habituel (classes transplantées, transports scolaires). La nouvelle politique éducative n'apparaît pas plus lorsque d'autres sujets, en rapport avec la jeunesse ou le scolaire, sont traités : création d'une permanence d'accueil, d'information et d'orientation pour les jeunes (CM, 22/11/1982), création d'un conseil communal de prévention de la délinquance (CM, 26/3/1985), signature d'une convention avec l'État pour le plan Informatique pour tous (CM, 14/10/1985), et tout au long de la période, signatures de conventions avec l'État pour la rénovation urbaine et la réhabilitation.

Le dépouillement du bulletin municipal, Aubervilliers, bulletin d'information mensuel (Am.) reflète un désintérêt progressif de la part de la municipalité. En 1982, suite à une journée d'étude sur la lutte contre les inégalités et les échecs scolaires réunissant des acteurs éducatifs et des élus, un article de quatre pages présente la démarche ZEP : "Quant aux moyens nouveaux, nous pensons là encore particulièrement à la création des zones d'éducation prioritaires (ZEP) à mettre en place là où les dégâts sont les plus graves, il doit s'agir de projets cohérents mis sur pied avec la participation [...] de tous ceux qui concourent à une meilleure qualité de vie scolaire. L'école doit jouer un grand rôle

\footnotetext{
${ }^{15}$ Ministre de la santé puis de l'emploi, il faisait donc partie du gouvernement de Pierre Mauroy au moment de l'implantation des ZP.

16 "Relevé de décisions concernant les zones d'éducation prioritaire», courrier du recteur aux chefs d'établissement, daté du $1^{\text {er }}$ mars 1982.

${ }^{17}$ Entretien du 2 décembre 2005.
} 
dans la mise en ouvre du changement tant attendu. Pour ce faire elle doit elle aussi changer $»^{18}$. En juin 1982, une délégation comprenant la députée et la maire-adjointe défend notamment auprès du cabinet du ministre l'ouverture d'une classe primaire «qui se trouve dans un secteur [...] (qui) soit considéré comme ZEP c'est-à-dire zone prioritaire ${ }^{19}$. En octobre 1982, l'article (non signé) «Les luttes ont payé », insiste sur "la restructuration profonde de l'ensemble du système scolaire qui est nécessaire. [...] Le rôle des enseignants est capital et ces derniers ne peuvent plus travailler comme il y a 20 ans [...] en revanche un enseignant préparé à travailler en équipe, à confronter sa discipline avec d'autres, à favoriser la participation d'intervenants nouveaux dans l'école (travailleurs spécialisés susceptibles d'apporter une information précise aux élèves intéressés), un enseignant aussi proche des préoccupations des jeunes en mesure de connaître leurs difficultés familiales et sociales, ouvert à toutes les innovations, constituerait un facteur primordial de l'évolution du système scolaire $»^{20}$. À partir de 1983, les propos sur l'ouverture de l'école sur la vie, la réduction des inégalités, la lutte contre l'échec scolaire, restent généraux, sans lien avec l'élaboration d'un projet et la rénovation des collèges, pourtant en cours.

Après une première période où des élus communistes se positionnent favorablement face à la création d'une ZP, ce sujet disparait ensuite du bulletin municipal, qui se contente de rapporter les informations habituelles : réjouissance et approbation lors des créations de postes, contestation commune entre élus, parents et enseignants lors des prévisions de fermeture de classes, délégations à l'inspection académique, courriers et parfois grèves. La municipalité continue de proposer des services et un accompagnement culturel et sportif aux enseignants ${ }^{21}$, tout en veillant périodiquement à rassurer les enseignants sur les initiatives municipales. Les élus communistes n'ont pas pris le risque de se brouiller localement avec les enseignants, voire d'entrer en conflit ouvert avec une partie de leur électorat. Créer une ZEP conduisait à s'interroger collectivement sur le fonctionnement des établissements, les pratiques pédagogiques, les relations avec les familles et les partenaires. A Aubervilliers, le choix de rester à l'écart de la politique d'éducation prioritaire jusqu'en 1998 serait ainsi une réponse pragmatique à un rapport de forces local et non une décision de principe débattue collectivement et officiellement.

\section{Le Havre : accompagner comme à l'ordinaire}

La ZEP du Havre Nord présente une singularité : elle a été la plus importante de France en effectifs d'élèves. En 1985, elle regroupe 5 collèges et 29 écoles de leur secteur, pour une population totale évaluée à 11000 élèves $^{22}$. Les quartiers concernés sont tous en politique de la ville ${ }^{23}$. Ce gigantisme ne parait pas être perçu comme un problème par le coordonnateur en poste de 1982 à 1999, ni par les

\footnotetext{
${ }^{18}$ Am., mars 1982, p. 4-7.

19 «Quelle rentrée à Aubervilliers ? », Am., juin 1982, p. 10-12. Christian Join-Lambert rapporte que des élus de Seine-Saint-Denis venaient plaider directement auprès du cabinet du ministre pour que davantage de zones prioritaires soient créées dans leur département et leurs communes (entretien du 21 janvier 2004).

${ }^{20}$ Am., octobre 1982, p. 4-6.

${ }^{21}$ Am., mars 1989, p. 11-12.

${ }^{22}$ Que se passe-t-il dans les zones prioritaires ? CRDP de Rouen, 1986. Il existe une seconde ZEP, celle du Havre Sud. Seul le centre-ville n'est pas classé en éducation prioritaire.

${ }^{23}$ La ville du Havre, qui a annexé entre 1953 et 1973 plusieurs communes ou parties de communes voisines, comporte plusieurs quartiers concernés par les programmes Habitat et vie sociale puis Développement social de quartier (DSQ) : Bléville, Caucriauville, Mont-Gaillard.
} 
élus $^{24}$. De fait, les archives attestent une dynamique certaine, un suivi et une régulation continus ${ }^{25}$. Communiste de 1965 à 1995, la ville permet d'observer un positionnement différent de celui d'Aubervilliers : ici, la municipalité s'est comportée vis-à-vis de la politique des ZEP comme à l'ordinaire, en soutenant les acteurs sans chercher à introduire ou catalyser des changements.

Les procès-verbaux de conseils municipaux (1981-1985) et les bulletins municipaux Le Havre information (1973-1999) ${ }^{26}$ témoignent du soutien des élus locaux à l'éducation prioritaire sous trois formes: les déclarations adressées aux autorités académiques, les moyens financiers accordés, la participation des élus en charge des secteurs scolaires aux réunions de la ZEP. Les élus majoritaires (PCF et PS) n'émettent jamais de critiques contre la politique d'Alain Savary et ils soutiennent les décisions prises par les responsables et le coordonnateur de la ZEP. Les relations de confiance entre enseignants et élus s'expliquent en partie par le choix du maire André Duroméa ${ }^{27}$ de confier l'enseignement à des adjoints professionnels de l'éducation. C'est le cas de Daniel Paul (PCF), instituteur, $3^{\mathrm{e}}$ adjoint, chargé des lycées et de l'enseignement professionnel, de la mission locale 16-25 ans, de la formation permanente et de l'université ; mais aussi à partir de 1983 de Bernard Cassagnabère, éducateur ${ }^{28}, 13^{\mathrm{e}}$ adjoint, chargé de l'enseignement primaire, des collèges et des classes de découverte.

Le journal municipal ne publie aucun article sur la politique d'éducation prioritaire ou sur ses projets de zone entre mai 1981 et juillet 1984. Il rend compte des visites de ministres (Mauroy, Ralite, Fiterman, Le Pensec), des travaux réalisés dans les écoles, du maintien des postes, de la mobilisation pour obtenir une université indépendante et un LEP supplémentaire. En revanche, lors des conseils municipaux (CM), l'évocation des ZEP et des PAE est liée, d'une part, au financement accordé par la ville aux projets $^{29}$, d'autre part, aux motions votées pour le maintien ou la création de postes d'enseignants $^{30}$. La politique ZEP aurait pu être évoquée explicitement à l'occasion d'autres débats, lors de la création de la mission locale pour les jeunes de 16 ans et plus, ou bien à propos de la formation professionnelle évoquée le 14 décembre 1982. Daniel Paul déclare alors : "[Au Havre] il y a environ 1500 jeunes, de 16 à 18 ans, [...] (avec) retards scolaires, sans diplôme, sans formation technique, sans emploi ». [...]. Il faut tarir la source qui est la cause d'une partie des échecs et des rejets du système scolaire [...]. Il convient, me semble-t-il, de travailler, dès à présent, sur un $2^{e}$, voire un $3^{e}$ LEP supplémentaire sur notre agglomération ». Alors même que la ville compte déjà 8 LEP, 2 lycées techniques et 3 lycées classiques et modernes (CM, 30 mars 1983). Si l'échec scolaire et le manque de qualification sont bien perçus comme des problèmes prégnants, les solutions imaginées par les élus n'ont pas anticipé ce qui sera l'objectif ministériel à partir de 1985, conduire $80 \%$ d'une classe d'âge «au niveau» du baccalauréat (général, technologique, professionnel), tant parait dominante dans leur esprit la seule formation professionnelle, socle de la culture ouvrière. La lutte

\footnotetext{
${ }^{24}$ C'est après la seconde relance des ZEP, en 1999, que la ZEP (8799 élèves alors) sera scindée en deux REPZEP, à la demande des inspecteurs et de deux principaux.

${ }^{25}$ Ces archives sont en cours de versement aux Archives départementales de Seine-Maritime.

${ }^{26}$ Archives municipales du Havre, cote PER 062. Désormais, Hinf.

${ }^{27}$ Né en 1917, André Duroméa est serrurier de profession. Élu maire en 1971, il cèdera son poste en 1994 un an avant le changement de majorité et l'élection d'Antoine Rufenacht (RPR).

${ }^{28}$ Ancien instituteur spécialisé exerçant en ZEP, il est ensuite éducateur à la PJJ, avant de devenir magistrat (2001), après avoir soutenu une thèse sur le droit des mineurs (1999).

${ }^{29}$ Par exemple, pour la seule année 1984, séances du conseil municipal du 27 février, 24 septembre, 5 novembre, 17 décembre.

${ }^{30}$ Séances du 17 mai 1982, 30 mai 1983, 25 juin 1984.
} 
Heurdier, L. \& Bongrand, P. (2021). Des villes communistes face à l'éducation prioritaire, entre engagement marqué et refus (1981-1984). Carrefours de l'éducation, 51, 53-65. https://doi.org/10.3917/cdle.051.0053

contre l'échec scolaire s'accompagne aussi de l'objectif «d'amener le plus loin possible le plus grand nombre d'élèves » ${ }^{31}$.

Un seul vrai débat relie ZEP et échec scolaire. Le 15 mai 1982, une motion salue la non fermeture de classes d'écoles de ZEP, approuve dans le même temps l'ouverture de classes spécialisées, la création de deux groupes d'aide psychopédagogique (GAPP), mais appelle à aller au-delà : «le remplacement des maîtres absents [...] ; le déblocage de moyens afin que [les ZEP] puissent réellement jouer leur rôle, et entraîner l'adhésion de ceux qui y ont participé pendant plusieurs semaines et plusieurs mois mais aussi et surtout de ceux qui n'y ont pas suffisamment participé ; la constitution d'équipes pédagogiques les plus larges possibles, ce qui nécessite bien évidemment que les concertations existent avec les équipes éducatives existant dans les quartiers, ce qui implique la création de postes supplémentaires [...] ». Un vœu est adopté à l'unanimité, après qu'un autre élu a souligné le sérieux des enseignants, responsables d'associations, équipes éducatives, réunis autour des projets des deux $\mathrm{ZEP}^{32}$.

Au Havre, la municipalité communiste a donc accompagné et soutenu le travail entrepris dans les ZEP, notant ce qui progresse, ce qui manque, sans parti pris marquant. La concordance apparente des aspirations entre les plus engagés des membres de l'Éducation nationale et les élus communistes évite tout conflit ou blocage dans la mise en œuvre entre 1981 et 1984.

\section{Orly : s'engager fermement pour faire des ZEP l'instrument du changement}

À Orly, la dynamique de la ZEP est activement portée par la municipalité. Bien avant 1981, de nombreuses actions d'accompagnement social et culturel accompagnent la lutte contre l'échec scolaire. L'ilot sensible du grand ensemble Orly-Choisy est objet du programme Habitat et vie sociale. En 1982, différentes politiques étatiques s'y déploient : commission nationale DSQ, création d'une mission locale 16-25 ans, mise en place de la ZEP qui regroupe 11 écoles et le collège Desnos, considéré comme l'un des deux plus difficiles du département ${ }^{33}$.

La ville soutient la création en janvier 1983 d'une équipe d'animation de la ZEP dont la responsable et coordonnatrice est une mère d'élève, Odette Terrade, qui assumera ce rôle jusqu'en 1989. Sollicitée alors qu'elle est présidente du conseil local FCPE à l'école Marcel Cachin (classée en ZEP), elle est aussi élue au conseil municipal en 1983, et nommée par Gaston Viens ${ }^{34}$, maire d'Orly, adjointe en charge du personnel communal ${ }^{35}$.

\footnotetext{
${ }^{31}$ Conférence de presse du recteur Bachelard à la rentrée 1984. Hinf $\mathrm{n}^{\circ}$ 83, septembre 1984.

${ }^{32}$ Le «Projet d'éducation prioritaire pour la zone Mare rouge, Mont-Gaillard, Bléville », établi par le coordonnateur de la ZEP du Havre nord, probablement au printemps 1982, présente de manière détaillée (46 pages) l'ensemble de la réflexion conduite par les « enseignants, parents d'élèves, conseillers d'orientation, membres de l'administration, assistantes sociales, élus locaux, éducateurs, associations de quartiers ».

${ }^{33}$ Les indicateurs de 1985-1986, sans grand changement depuis 1983, font état de $61 \%$ de CSP défavorisées, $41 \%$ d'élèves non francophones, $45 \%$ de boursiers, $25 \%$ d'élèves en retard de deux ans et plus en 6 . «Classement des collèges du Val-de-Marne », Archives départementales du Val-de-Marne (AD 94), 4304 W 4.

${ }^{34}$ Ancien résistant et déporté, Gaston Viens (1924-2015) est maire de 1965 à 2009. Communiste depuis 1941, il est exclu du parti en 1989 en tant que « reconstructeur ».

${ }^{35}$ Elue présidente de la FCPE du Val-de-Marne en juin 1985, elle adhère au PCF en 1986. Des conflits internes conduiront à son éviction de toute responsabilité municipale en 1989. Elle poursuivra sa carrière politique comme sénatrice communiste (1997-2004, puis 2007-2011). (Entretien du 22 mars 2017)
} 
La création, le fonctionnement et les spécificités pédagogiques de cette ZEP sont très bien décrits dans deux ouvrages contemporains (CRESAS, 1983, 1985). Pour examiner les formes et mobiles de l'implication des élus communistes, la source principale est le journal municipal mensuel, le Nouveau Journal d'Orly $(\mathrm{NJO})^{36}$, entre 1981 et 1985.

D'emblée, l'importance accordée à l'éducation et à la ZEP est frappante : en plus des articles classiques d'actualité scolaire municipale (travaux dans les écoles, classes de découverte, activités péri-éducatives...), de nombreuses interviews d'enseignants et de parents d'élèves paraissent régulièrement. Les titres choisis marquent le soutien à la politique d'Alain Savary : «Rentrée : un souffle nouveau » $\left(N J O \mathrm{n}^{\circ}\right.$ 55, août 1981); "Changer l'école I : le CES Desnos » (article où quatre représentants syndicaux et quatre représentants des parents d'élèves donnent leurs points de vue, convergents, sur l'échec scolaire et les nécessaires transformations de l'école) ${ }^{37}$; «L'école en mouvement $\gg\left(N J O \mathrm{n}^{\circ}\right.$ 73, mai 1983). L'ensemble des acteurs locaux réclament néanmoins des moyens, en particulier de remplacement. Des parents souhaitent «seulement que les enfants des cités ouvrières d'Orly disposent des mêmes ouvertures culturelles que ceux de Saint-Mandé ou du $16^{e}$ arrondissement $»^{38}$. Avant sa nomination, Odette Terrade revendique une place pour les parents : «Notre démarche est constructive [...] nous cherchons à participer à égalité avec les autres composantes de la concertation. Nous ne voulons pas assumer un rôle simplement consultatif. À notre avis, c'est là un excellent moyen d'ouvrir l'école sur la société, la vie »"

Les frais de fonctionnement de l'équipe d'animation sont pris en charge par la mairie, qui met en outre à disposition ses salles pour les réunions qui ont lieu très régulièrement. Cette force d'appui municipale est au service de la dynamique sociale qui s'ébauche. L'équipe d'animation se fixe trois missions : coordonner, suivre et valoriser les actions. Des commissions de travail sont créées. Le maire-adjoint à l'enseignement déclare en août 1983: "Les réformes ne remplaceront jamais l'élément moteur de toute transformation: l'intervention de tous et de chacun, sur une voie définie collectivement. [...] coordonner les activités extrascolaires avec celles qui se déroulent dans l'établissement. La réforme Savary concernant les collèges nous indique un chemin à prospecter, de façon à éviter toute sélection précoce ou hâtive $»^{40}$. En 1984 et 1985, c'est l'équipe du Centre de recherche de l'éducation spécialisée et de l'adaptation scolaire (CRESAS) qui est mise en avant. Cette dynamique est soutenue par la présence d'inspecteurs départementaux de l'Éducation nationale mobilisés en lien avec l'école normale de Bonneuil ${ }^{41}$. Au fil des années, de nouveaux acteurs entrent dans le processus de transformation des pratiques - sans que soient pour autant niés ou levés tous les obstacles et les résistances : la mobilité des professeurs du CES, l'insuffisante participation des parents à «l'élaboration et à la mise en cuvre des projets, [...] la difficulté de se faire reconnaitre comme partenaire à part entière $»^{42}$.

La ville communiste d'Orly s'est fortement engagée dans la nouvelle politique éducative qui répondait aux attentes des élus comme des représentants FCPE, avec le soutien d'acteurs éducatifs dont des

\footnotetext{
${ }^{36}$ Collection consultée aux AD 94.

${ }^{37}$ «Les ZEP sont pour nous un moyen de débuter les changements sans trop attendre [...] Les changements à réaliser sont multiples et ne s'imposeront pas d'eux-mêmes. Je pense par exemple aux mentalités qu'il faut changer ». NJO, $\mathrm{n}^{\circ} 58$, décembre 1981, p. 24.

${ }^{38} N J O, \mathrm{n}^{\circ} 59$, janvier 1982, p. 24.

${ }^{39} \mathrm{NJO}, \mathrm{n}^{\circ} 66$, octobre $1982, \mathrm{p} .31$.

${ }^{40} \mathrm{NJO}, \mathrm{n}^{\circ} 75$, août 1983 , p. 12.

${ }^{41}$ Le directeur, M. Gilbert-Collet, est l'époux de l'inspectrice de maternelle investie sur Orly.

${ }^{42}$ NJO, n ${ }^{\circ} 84$, juin 1984, p. 9.
} 
enseignants engagés dans une transformation de pratiques pédagogiques. Le journal municipal diffusé auprès de toute la population s'est fait le relais de la dynamique locale interinstitutionnelle en œuvre, diffusant régulièrement des informations sur la ZEP, les avancées mais aussi les obstacles rencontrés.

Malgré une ligne nationale favorable aux ZEP, les municipalités communistes s'impliquent inégalement dans la mise en œuvre de cette politique. Les relations entre élus et enseignants, qui sont en partie liées aux rapports de force politiques et syndicaux locaux, paraissent influencer davantage la réception locale de l'éducation prioritaire que les discours officiels du PCF. Ce parti, inégalement influent dans les organisations syndicales, n'était probablement pas en mesure de défendre une politique partenariale et interdegrés. D'autant que le PCF entre alors dans un processus de redéfinition du rôle des élus, qui gagnent en autonomie et en pouvoir au sein de l'organisation. L'éducation prioritaire est un bon exemple des «pluralités de formes » prises par le communisme français dans les territoires (Bellanger \& Mischi, 2014).

\section{Bibliographie}

Bellanger E., Mischi J. (dir.) (2014). Les territoires du communisme. Élus locaux, politiques publiques et sociabilités militantes. Paris : Armand Colin.

Bongrand P. (2009). La scolarisation des mours. Socio-histoire de deux politiques de scolarisation, en France, depuis la Libération. Thèse de science politique, Université de Picardie, 2009.

Bongrand P. (2013). La gauche contre la démocratisation de l'enseignement ? À la recherche des oppositions communistes à la politique des zones d'éducation prioritaires. Prolégomènes à une enquête. AREF - Actualités de la recherche en éducation et formation, Montpellier. https://hal.archives-ouvertes.fr/hal-03184218

Bourgarel A., Join-Lambert C., Bablet M. (2006). L'aventure des ZEP. In F. Pavé (éd.). La modernisation silencieuse des services publics. Paris : L'Harmattan. p. 43-53.

CRESAS (1983). Écoles en transformation: zones prioritaires et autres quartiers. Paris: L'Harmattan/INRP.

CRESAS (1985). Depuis 1981, l'école pour tous? Zones d'éducation prioritaires. Paris: L'Harmattan/INRP.

Heurdier L. (2008). Vingt ans de politique d'éducation prioritaire dans trois départements français. Thèse de Sciences de l'éducation, Université Paris V.

Heurdier L. (2014). La politique d'éducation prioritaire. Un projet politique conduit hors du champ politique (1981-2001). Vingtième siècle, $\mathrm{n}^{\circ} 124$, p. 155-168.

Kherroubi M., Rochex J.-Y. (2002). La recherche en éducation et les ZEP en France. 1. Politique ZEP, objets, postures et orientations de recherche. Revue française de pédagogie, ${ }^{\circ}$ 140, p. 103-132.

Llobet, A. (2012). La neutralisation de la réforme de l'éducation prioritaire. Un collège face au dispositif «ambition-réussite ». Gouvernement et action publique, $\mathrm{n}^{\circ}$ 3, p. 77-99.

Prost A. (2013). Du changement dans l'école. Paris : Éditions du Seuil.

Robert A. (2002). Syndicats et ZEP : d'une controverse implicite sur l'idée de justice à un consensus mou. Revue française de pédagogie, $\mathrm{n}^{\circ}$ 140, p. 21-31.

Robert B. (2009). Les politiques d'éducation prioritaire. Les défis de la réforme. Paris : PUF. 